\title{
Troponin-I Level After Major Noncardiac Surgery and Its Association With Long-Term Mortality
}

\section{A Retrospective Cohort With a 7-Year Follow-Up Period}

\author{
Minkwan KIm, ${ }^{1}$ MD, Minkook Son, ${ }^{1}$ MD, Dong Hyun LeE, ${ }^{1}$ MD, \\ Kyungil PARK, ${ }^{1,2} \mathrm{MD}$, and Tae-Ho PARK, ${ }^{1,2} \mathrm{MD}$
}

\begin{abstract}
SUMMARY
Individuals with intermediate to high cardiac risk for major noncardiac surgery suffer from perioperative myocardial ischemic injury. The purpose of this study was to evaluate the long-term impact of postoperative cardiac troponin elevation on clinical outcome after major noncardiac surgery.

Patients $(n=750)$ aged $\geq 50$ years who underwent major noncardiac surgery were eligible for the study. Postoperative cardiac troponin-I data were collected retrospectively and consecutively. The primary outcome measure was allcause mortality. The median follow-up period was 1727 days in all patients.

Among 750 patients, 92 (12.2\%) showed elevated postoperative troponin-I above $0.10 \mathrm{ng} / \mathrm{mL}$. Operative mortality was $4.1 \%$ (31 subjects), and patients with troponin-I elevation showed a higher operative mortality rate (RR: $4.23,95 \%$ CI: $2.67-11.31, P<0.001)$. In multivariate Cox regression analysis, a troponin-I concentration above $0.10 \mathrm{ng} / \mathrm{mL} \mathrm{was}$ associated with all-cause mortality (RR: $1.73,95 \%$ CI: $1.27-2.36, P<0.001)$. It should be noted that there was a significant difference between patients with elevated and non-elevated troponin-I in the rate of mortality until 6 months. However, these differences disappeared after 6 months.

An elevated troponin-I level conferred an increase in mortality during the 7 year follow-up period after major noncardiac surgery. This difference in mortality was mainly derived from the result within the first 6 months. (Int Heart $\mathrm{J}$ 2016; 57: 278-284)
\end{abstract}

Key words: Myocardial ischemia, Death, Surgical procedures

A 11 surgical procedures elicit a stress response, although the extent of the response depends on the extent of the surgery and the use of anesthetics and analgesics that are used to reduce the response. The stress response can lead to increases in heart rate and blood pressure, which can precipitate episodes of myocardial ischemia. ${ }^{1)}$

Individuals with intermediate to high cardiac risk for noncardiac surgery suffer from perioperative myocardial ischemic injury. ${ }^{2)}$ Cardiac troponins are a useful tool that help clinicians in making the diagnosis of perioperative myocardial infarction. $^{3-5)}$ Many previous studies have investigated the role of cardiac troponins in the diagnosis of perioperative myocardial ischemic events and demonstrated the prognostic value of postoperative cardiac troponin elevation. ${ }^{6-9)}$ A recent metaanalysis of studies in predominantly high-risk surgery patients also showed that cardiac troponin elevation was a strong independent predictor of mortality within the first year after surgery. ${ }^{10)}$

\begin{abstract}
Editorial p.265
The development of perioperative cardiovascular complications not only affects the short-term period prognosis but may also influence the long-term consequences. However, few studies have explored the long-term, defined as beyond the first year after major noncardiac surgery, prognostic value of cardiac troponin elevation in the prediction of postoperative outcome. We undertook this study to evaluate the clinical outcome of cardiac troponin elevation after major noncardiac surgery. The present study extended the follow-up period to 7 years and attempted to obtain valuable data regarding longer-term mortality.
\end{abstract}

\section{MeTHODS}

Study populations: Patients aged $\geq 50$ years who underwent major noncardiac surgery at Dong-A University Medical Cent-

From the ${ }^{1}$ Regional Cardiocerebrovascular Center, Dong-A University Hospital and ${ }^{2}$ Division of Cardiology, Department of Internal Medicine, Dong-A University College of Medicine, Busan, South Korea.

This study was supported by grants from the Dong-A University Research fund.

Address for correspondence: Kyungil Park, MD, Regional Cardiovascular Center, Dong-A University Hospital, Division of Cardiology, Department of Internal Medicine, Dong-A University College of Medicine, 1-3 Ga, Dongdaesin-Dong, Seo-Gu, Busan, 602-714, South Korea. E-mail: cardiopark@ gmail.com, sotier@ dau.ac.kr Received for publication September 7, 2015. Revised and accepted November 17, 2015.

Released in advance online on J-STAGE April 28, 2016.

All rights reserved by the International Heart Journal Association. 
er from January 1, 2005 to December 31, 2007 were eligible for the study. Patients with preexisting troponin-I elevations were excluded, because this study aimed to evaluate the longterm clinical impact of post-operative troponin elevation. Daycase surgical procedures were considered to be of very low risk and postprocedural troponin-I values were unavailable. Thus, patients with day-case surgery were not included. The present study was approved by the institutional review board, which allowed the use of clinical data for this study.

Data collection: Data were collected retrospectively and consecutively. All data input was done by database managers and independent research nurses who were not aware of the study and had not participated in the management and care of the study patients. Clinical characteristics, cardiovascular risk factors, and medication on admission were documented. Data quality was assessed by reviewing a random sample of $10 \%$ of all medical records. All reported events were confirmed by reviewing hospital charts or by contacting the physicians. Follow-up was conducted in all patients using medical records or telephone interviews. To improve the completeness of followup, death data of all patients in this study were collected from the National Statistical Office of Korea.

Measurements: Troponin-I levels were collected from laboratory results. Study participants who consented to major noncardiac surgery underwent pre- and postoperative serum enzyme sampling. Troponin-I concentrations were measured before and at day 1 and 2 after major noncardiac surgery. In addition, serial measurements of troponin-I were also performed if clinical events were suspicious for myocardial ischemia or troponin-I of the first 2 days after noncardiac surgery was elevated. Troponin-I was measured using an Abbott AxSYM analyzer (Abbott Laboratories, Abbott Park, IL, USA) by mass immunoassay with the normal upper limit of $0.10 \mathrm{ng} / \mathrm{mL}$ specified by the manufacturer. According to the manufacturer's recommendations and based on our analyses, troponin positive was defined as a troponin-I level higher than $0.10 \mathrm{ng} / \mathrm{mL}$. The study population was classified into troponin positive $(\mathrm{TnP})$ and negative $(\mathrm{TnN})$ groups according to the troponin-I level.

Study endpoint and definitions: The primary endpoint was allcause death at any point during the follow-up period. We defined all-cause death as death from any cause during followup. Operative mortality was defined as death occurring during hospitalization or within 30 days of surgery. Perioperative MI was defined according to the universal definition of MI., ${ }^{8,11}$ Major noncardiac surgery was defined by an expected hospital length of stay of at least 2 days, ${ }^{8,12}$ and one of the following: abdominal-aortic surgery, peripheral vascular surgery, laparotomy including abdominal and urogenital operations, major orthopedic surgery of the hip or spinal column, and thoracotomy. The study population was classified into vascular, included abdominal-aortic surgery and peripheral vascular surgery, and non-vascular surgery groups according to the type of surgery. Diabetes mellitus (DM) was defined as a history of diabetes, a fasting plasma glucose concentration above $126 \mathrm{mg} / \mathrm{dL}$, or the presence of therapy. Dyslipidemia and hypertension were defined as a documented history of dyslipidemia or high blood pressure requiring diet or medical therapy, respectively. Chronic kidney disease $(\mathrm{CKD})$ was defined as estimated glomerular filtration rate $(\mathrm{eGFR})<60 \mathrm{~mL} /$ minute per $1.73 \mathrm{~m}^{2}$ using the modification of diet in renal disease (MDRD) formula or se- rum creatinine $(\mathrm{Cr})>1.5 \mathrm{mg} / \mathrm{dL}$. The MDRD formula was defined as follows:

$$
\mathrm{eGFR}=186 \times \text { serum } \mathrm{Cr}^{-1.154} \times \mathrm{Age}^{-0.203} \times(0.742 \text { if fe- }
$$
male) where $\mathrm{Cr}$ concentration is in $\mathrm{mg} / \mathrm{dL}$.

Statistical analysis: Continuous variables are expressed as the mean $\pm \mathrm{SD}$ and were analyzed by the independent $t$-test to compare the differences with the control group. Categorical variables were compared by the chi-square test or Fisher exact test. To assess the cut-off values of the independent predictors of surgical outcomes, receiver-operating characteristic (ROC) curve analysis was used. The cut-off values were selected as one with the maximal sum of sensitivity and specificity. C-statistics analysis was performed to examine the independent prediction of troponin-I level when compared with the incorporation of other clinical variables. Baseline characteristics associated with all-cause death were estimated with univariate Cox regression models and reported as relative risk (RR) with the $95 \%$ confidence interval (CI). Those with a $P$ value $\leq 0.05$ were used in the multivariate Cox regression model to identify independent predictors for all-cause mortality. Variables that were identified to have statistical significance in multivariate regression underwent Kaplan-Meier survival analysis and were then compared with the log-rank test. Univariate and multivariate logistic regression models were used to define predictors of troponin positivity. All potential preoperative predictors with $P$ $<0.1$ on univariate logistic analysis were included in the multivariate logistic regression analysis, and a backward conditional selection method was used for variable selection by the model. In a supplementary analysis, we investigated the dose-response relation between cardiac troponin-I and 6-month mortality by stratifying peak cardiac troponin-I concentrations into 4 groups: $<0.10 \mathrm{ng} / \mathrm{mL}, 0.10$ to $1.00 \mathrm{ng} / \mathrm{mL}, 1.10$ to 2.00 , and $>$ $2.10 \mathrm{ng} / \mathrm{mL}$. The $<0.10 \mathrm{ng} / \mathrm{mL}$ group was the reference category to which the other groups were compared. Statistical analysis was performed using IBM SPSS version 21.0 for Windows (SPSS Inc., Chicago, IL, USA). All statistical tests were carried out at the 0.05 significance level.

\section{Results}

Baseline characteristics: A total of 750 subjects who underwent major noncardiac surgery were included. Baseline characteristics of the study population are presented in Table I. The proportion of men and DM patients was 405 patients (54.0\%) and 208 patients $(27.7 \%)$, respectively. The median follow-up was 1727 days (interquartile range: 663 to 2269 days). There were $92(12.2 \%)$ patients in the $\mathrm{TnP}$ group. Univariate analysis showed that $\mathrm{CKD}(P<0.001)$, heart failure $(P=0.007)$, previous PCI $(P=0.002)$, previous MI $(P<0.001)$, and vascular surgery $(P<0.001)$ were more frequent in the TnP group. Compared with the $\mathrm{TnN}$ group, patients in the $\mathrm{TnP}$ group showed a lower ejection fraction $(P=0.032)$. When clinical variables were taken into consideration, CKD (RR: $3.71,95 \%$ CI: 2.35-5.86, $P<0.001$ ), history of previous MI (RR: 2.25, 95\% CI: $1.35-3.72, P=0.002$ ), and vascular surgery (RR: 1.96, 95\% CI: 1.18-3.24, $P=0.009)$ emerged as independent predictors of troponin positivity in multivariate logistic regression analysis.

Clinical outcomes: In all patients, all-cause death occurred in 288 subjects $(38.4 \%)$ during the 7-year follow-up. Among 
Table I. Comparison of Baseline Clinical Characteristics Between Troponin Positive Group and Troponin Negative Group

\begin{tabular}{|c|c|c|c|c|}
\hline Variables & $\begin{array}{l}\text { Overall population } \\
\quad(n=750)\end{array}$ & $\begin{array}{l}\text { TnP group } \\
(n=92)\end{array}$ & $\begin{array}{l}\text { TnN group } \\
(n=658)\end{array}$ & $P$ \\
\hline \multicolumn{5}{|l|}{ Clinical variables } \\
\hline Age (years) & $66.79( \pm 8.6)$ & $68.2( \pm 8.9)$ & $66.6( \pm 8.6)$ & 0.103 \\
\hline Male - No. (\%) & $405(54.0)$ & $1.47( \pm 0.5)$ & $1.46( \pm 0.5)$ & 0.880 \\
\hline Hypertension - No. (\%) & $233(31.1)$ & $33(35.9)$ & $200(30.4)$ & 0.288 \\
\hline Diabetes mellitus - No. (\%) & $208(27.7)$ & $32(34.8)$ & $176(26.7)$ & 0.107 \\
\hline Chronic kidney disease - No. (\%) & $98(13.1)$ & $32(34.8)$ & $66(10.0)$ & $<0.001$ \\
\hline Dyslipidemia - No. (\%) & $46(6.1)$ & $5(5.4)$ & $41(6.2)$ & 0.766 \\
\hline Atrial fibrillation - No. (\%) & $53(7.1)$ & $7(7.6)$ & $46(7.0)$ & 0.829 \\
\hline Heart failure - No. (\%) & $49(6.5)$ & $12(13.0)$ & $37(5.6)$ & 0.007 \\
\hline Previous MI - No. (\%) & $76(10.1)$ & $22(23.9)$ & $64(8.2)$ & $<0.001$ \\
\hline Previous stroke - No. (\%) & $28(3.7)$ & $5(5.4)$ & $23(3.5)$ & 0.358 \\
\hline Previous CABG - No. (\%) & 12 & $1(1.1)$ & $12(1.8)$ & 0.513 \\
\hline Previous PCI - No. (\%) & 45 & $12(13.0)$ & $33(5.0)$ & 0.002 \\
\hline Ejection fraction $(\%)$ & 58.8 & 57.0 & 59.1 & 0.032 \\
\hline Heart rate (beats/minute) & $78.2( \pm 9.4)$ & $81.5( \pm 12.5)$ & $77.8( \pm 8.9)$ & 0.007 \\
\hline Systolic blood pressure (mmHg) & $121.2( \pm 15.5)$ & $121.6( \pm 18.1)$ & $121.2( \pm 15.2)$ & 0.799 \\
\hline Distolic blood pressure (mmHg) & $76.7( \pm 9.3)$ & $77.2( \pm 11.8)$ & $76.6( \pm 8.9)$ & 0.602 \\
\hline \multicolumn{5}{|l|}{ Medications - No. (\%) } \\
\hline Anti-platelet agent & $152(20.3)$ & $30(32.6)$ & $122(18.5)$ & 0.002 \\
\hline Loop diuretic & $48(6.4)$ & $7(7.6)$ & $41(6.2)$ & 0.613 \\
\hline Beta-blocker & $118(15.7)$ & 19 (20.7) & $99(15.0)$ & 0.167 \\
\hline ACEi & $133(17.7)$ & $23(25.0)$ & $110(16.7)$ & 0.051 \\
\hline $\mathrm{CCB}$ & $163(21.7)$ & $23(25.0)$ & $140(21.3)$ & 0.615 \\
\hline Statin & $56(7.5)$ & $7(7.6)$ & $49(7.4)$ & 0.956 \\
\hline \multicolumn{5}{|l|}{ Laboratory findings } \\
\hline Hemoglobin (g/dL) & $12.1( \pm 2.0)$ & $11.8( \pm 2.4)$ & $12.2( \pm 1.9)$ & 0.082 \\
\hline Platelet $\left(\times 10^{3} / \mathrm{mm}^{3}\right)$ & $247.6( \pm 92.1)$ & $242.0( \pm 84.8)$ & $248.4( \pm 93.1)$ & 0.499 \\
\hline Urea nitrogen $(\mathrm{mg} / \mathrm{dL})$ & $20.8( \pm 15.1)$ & $29.2( \pm 23.3)$ & $19.6( \pm 13.1)$ & $<0.001$ \\
\hline Serum creatinine $(\mathrm{mg} / \mathrm{dL})$ & $1.4( \pm 1.6)$ & $2.5( \pm 3.0)$ & $1.2( \pm 1.3)$ & $<0.001$ \\
\hline Protein (g/dL) & $6.5( \pm 0.9)$ & $6.4( \pm 1.0)$ & $6.6( \pm 0.9)$ & 0.060 \\
\hline Albumin $(\mathrm{g} / \mathrm{dL})$ & $3.8( \pm 0.6)$ & $3.7( \pm 0.6)$ & $3.8( \pm 0.6)$ & 0.007 \\
\hline Total cholesterol (mg/dL) & $176.1( \pm 43.8)$ & $164.3( \pm 47.8)$ & $177.8( \pm 43.0)$ & 0.006 \\
\hline Postoperative troponin-I (ng/mL) & $0.31( \pm 1.89)$ & $2.52( \pm 4.86)$ & $0.01( \pm 0.02)$ & $<0.001$ \\
\hline \multicolumn{5}{|l|}{ Surgical kind - No. (\%) } \\
\hline \multicolumn{5}{|l|}{ Vascular surgery } \\
\hline Abdominal-aortic surgery & $31(4.1)$ & $9(9.8)$ & $22(3.3)$ & 0.004 \\
\hline Peripheral vascular surgery & $52(6.9)$ & $15(16.3)$ & $37(5.6)$ & $<0.001$ \\
\hline \multicolumn{5}{|l|}{ Non-vascular surgery } \\
\hline Laparotomy & $434(57.9)$ & $50(54.3)$ & $384(58.4)$ & 0.466 \\
\hline Major orthopedic surgery & $213(28.4)$ & $14(15.2)$ & $199(30.2)$ & 0.003 \\
\hline Thoracotomy & $20(2.7)$ & $4(4.3)$ & $16(2.4)$ & 0.285 \\
\hline \multicolumn{5}{|l|}{ Anesthesia kind - No. (\%) } \\
\hline General & $600(80.0)$ & $63(68.5)$ & 537 (81.6) & 0.428 \\
\hline Local & $131(17.5)$ & $24(26.1)$ & $107(16.3)$ & 0.321 \\
\hline Combine (local to general) & $2(0.3)$ & $0(0.0)$ & $2(0.3)$ & - \\
\hline Spinal & $17(2.2)$ & $5(5.4)$ & $12(1.8)$ & 0.584 \\
\hline
\end{tabular}

ACEi indicates angiotensin-converting-enzyme inhibitor; CCB, calcium channel blocker; MI, myocardial infarction; TnN, troponin negative; and $\mathrm{TnP}$, troponin positive.

these 288 patients, 50 in the TnP group died and thus all-cause mortality was estimated to be $54.3 \%$. Among the patients in the TnN group, 238 died during the follow-up period, and allcause mortality was estimated to be $36.2 \%$. Operative mortality was $4.1 \%$ (31 subjects) and the incidence of perioperative MI was $3.1 \%$ (23 subjects) in the overall population. Of these 23 patients, 16 were in the $\mathrm{TnP}$ group and 7 were in the $\mathrm{TnN}$ group. The TnP group had a higher prevalence of perioperative MI $(P<0.001)$ and a higher mortality rate $(P<0.001)$ than the $\mathrm{TnN}$ group. Subjects with perioperative MI had a higher longterm mortality rate (RR: $4.28,95 \% \mathrm{CI}: 1.23-15.42, P=0.026)$. The TnP group had a higher operative mortality rate (RR: 4.23, 95\% CI: $2.67-11.31, P<0.001)$. In the follow-up death group,
46 subjects $(55.4 \%$ of 83$)$ were in the vascular surgery group and 242 subjects (36.3\% of 667) were in the non-vascular surgery group. The vascular surgery group had a higher mortality rate than the nonvascular group and the difference was statistically significant (RR: $2.18,95 \%$ CI: $1.38-3.46, P=0.001$ ). Operative mortality was not significantly different between the vascular and non-vascular groups (RR: 1.67, 95\% CI: 0.94$2.98, P=0.078)$. Eleven subjects $(1.5 \%)$ had perioperative MI. The incidence of perioperative MI was higher in the vascular surgery group (RR: 7.06, 95\% CI: $2.11-23.68, P=0.004$ ).

Independent predictors of all-cause death in major noncardiac surgery: In univariate Cox regression analysis, age, sex, DM, CKD, previous MI, previous stroke, ejection fraction, use of 
Table II. Univariate and Multivariate Cox Regression Analysis of Predictors of All-Cause Death

\begin{tabular}{lcrrr}
\hline & \multicolumn{1}{c}{ Univariate analysis } & \multicolumn{2}{c}{ Multivariate analysis } \\
& RR $(95 \% \mathrm{CI})$ & $P$ & $\mathrm{RR}(95 \% \mathrm{CI})$ & $P$ \\
\hline Age (over 70 years) & $1.57(1.23-1.99)$ & $<0.001$ & $1.62(1.28-2.04)$ & $<0.001$ \\
Male & $1.56(1.22-2.03)$ & 0.001 & $1.67(1.31-2.13)$ & $<0.001$ \\
Diabetes mellitus & $2.12(1.10-4.30)$ & 0.011 & $1.12(0.86-1.45)$ & 0.390 \\
Chronic kidney disease & $2.46(1.32-3.91)$ & $<0.001$ & $1.14(0.81-1.59)$ & 0.460 \\
Previous MI & $2.38(1.24-4.97)$ & 0.038 & $1.87(1.31-2.84)$ & 0.511 \\
Previous stroke & $2.66(1.59-5.87)$ & 0.023 & $1.48(0.88-2.49)$ & 0.143 \\
Ejection fraction $(<50 \%)$ & $1.92(1.26-2.93)$ & 0.003 & $2.10(1.45-3.04)$ & $<0.001$ \\
Loop diuretics & $2.56(1.20-4.562)$ & 0.010 & $1.34(0.87-2.06)$ & 0.179 \\
Dyslipidemia & $0.69(0.48-0.94)$ & 0.011 & $0.89(0.68-1.17)$ & 0.401 \\
Heart rate $\geq 80$ beats/minute & $2.12(1.28-3.49)$ & $<0.001$ & $1.49(1.17-1.88)$ & 0.001 \\
Hemoglobin $(<12 \mathrm{~g} / \mathrm{dL})$ & $2.92(1.97-4.49)$ & $<0.001$ & $1.78(1.40-2.26)$ & $<0.001$ \\
Troponin positivity & $2.56(1.93-3.76)$ & $<0.001$ & $1.73(1.27-2.36)$ & $<0.001$ \\
Vascular surgery & $2.31(1.38-3.76)$ & 0.001 & $1.02(0.71-1.47)$ & 0.901 \\
\hline
\end{tabular}

MI indicates myocardial infarction.

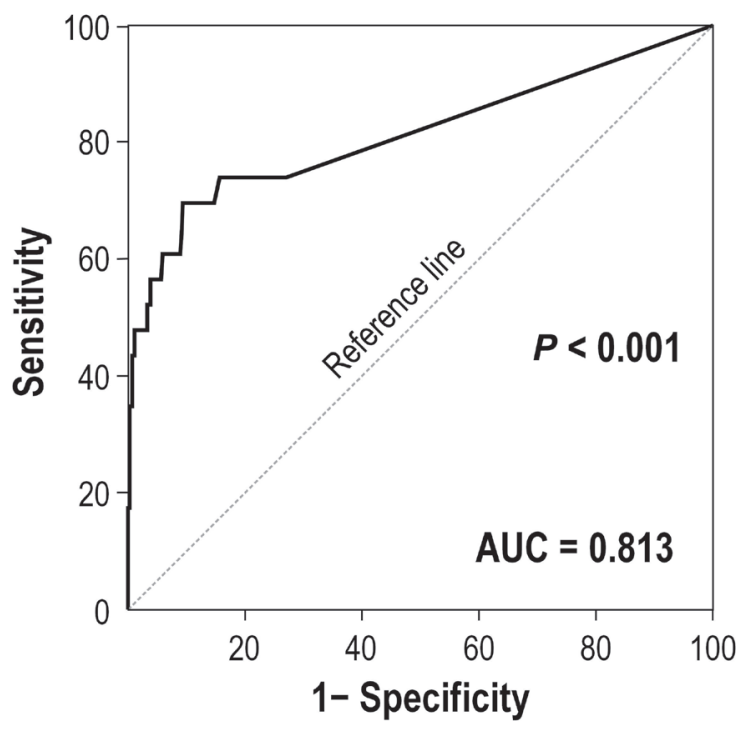

Figure 1. ROC curve for postoperative troponin-I for prediction of allcause mortality. The dotted line represents the non-discrimination line. The AUC is 0.813 (95\% CI: $0.69-0.93, P<0.001$ ). ROC indicates receiver-operating characteristic; and AUC, area under curve.

loop diuretics, dyslipidemia, heart rate, hemoglobin, vascular surgery, and $\mathrm{TnP}$ were related to all-cause death. To determine independent factors related to all-cause death, we performed multivariate Cox regression analysis (Table II). In the multivariate Cox regression, age (RR: 1.62, 95\% CI: 1.28-2.04, $P<$ 0.001 ), male gender (RR: $1.67,95 \%$ CI: $1.31-2.13, P<0.001)$, ejection fraction (RR: $2.10,95 \% \mathrm{CI}: 1.45-3.04, P<0.001$ ), heart rate (RR: $1.49,95 \% \mathrm{CI}: 1.17-1.88, P=0.001)$, hemoglobin (RR: $1.78,95 \%$ CI: $1.40-2.26, P<0.001$ ), and TnP (RR: 1.73 , 95\% CI: $1.27-2.36, P<0.001)$ were independent predictors of all-cause death. Elevated troponin-I was associated with death in both univariate and multivariate analyses. In the ROC curve, the elevation of postoperative troponin-I above $0.1 \mathrm{ng} / \mathrm{mL}$ was the point of both the highest sensitivity and specificity to predict long-term mortality (Figure 1). The mean event-free survival rate estimated by Kaplan-Meier analysis

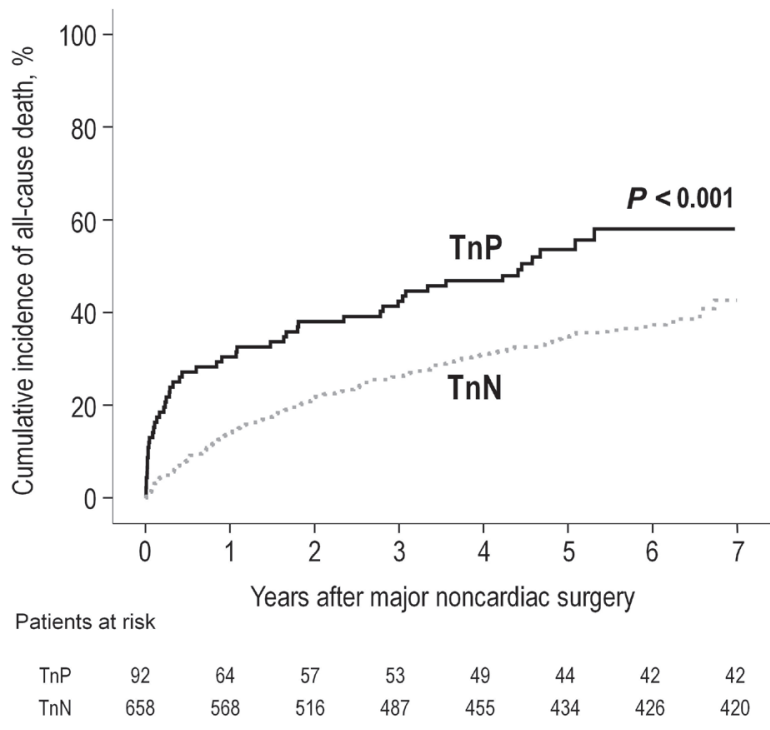

Figure 2. Seven-year cumulative incidence of all-cause death in patients in troponin-I positive and negative groups. Numbers of patients at risk are shown below each graph. Abbreviations as in Table II.

was longer in the TnN group $(P<0.001)$ (Figure 2). Such differences in long-term mortality during the 7-year follow-up period between the two groups were derived mainly from the results within the first 6 months by landmark analysis. Within the first 6 months, a difference in the all-cause death rate was observed between the $\mathrm{TnP}$ and $\mathrm{TnN}$ groups $(27.1 \%$ versus $8.5 \%$, RR: $1.63, P<0.001$ at Log Rank) (Figure 3). However, there was no difference in the rate of all-cause death $(P=$ 0.102 ) from the survival curve beyond 6 months (Figure 3 ).

In each ROC analysis for the other independent factors, we found that a heart rate above 80 beats per minute (sensitivity $51.4 \%$, specificity $63.2 \%, P<0.001$, area under the curve $=$ 0.58 ), age above 70 years (sensitivity $53.8 \%$, specificity $62.1 \%, P<0.001$, area under the curve $=0.61$ ), and ejection fraction below $50 \%$ (sensitivity $86.5 \%$, specificity $11.9 \%, P=$ 0.020 , area under the curve $=0.45$ ) effectively predicted all- 


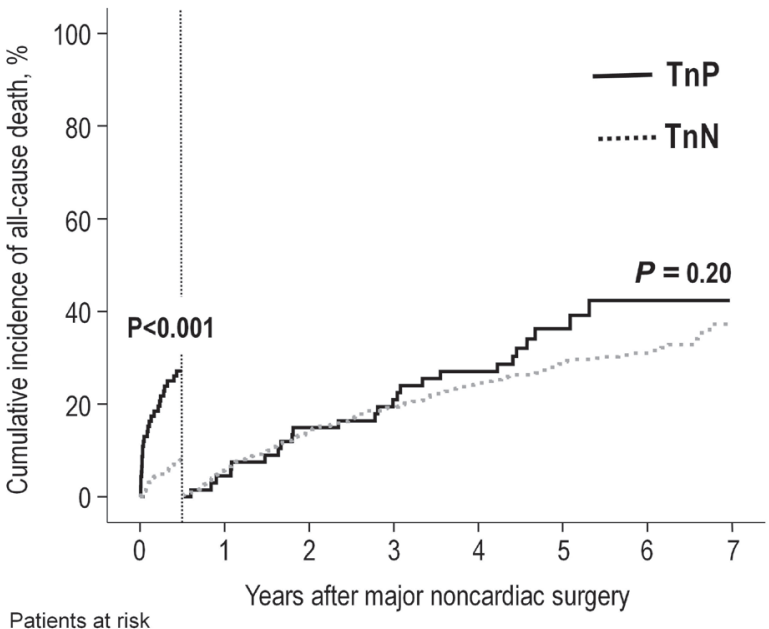

$\begin{array}{ccccccccc}\mathrm{TnP} & 92 & 64 & 57 & 53 & 49 & 44 & 42 & 42 \\ \mathrm{TnN} & 658 & 568 & 516 & 487 & 455 & 434 & 426 & 420\end{array}$

Figure 3. Landmark analysis of study population. Cumulative risk of allcause death during the first 6 months and thereafter for patients with troponin-I positive $(>0.10 \mathrm{ng} / \mathrm{mL})$ versus negative $(<0.10 \mathrm{ng} / \mathrm{mL})$ level Numbers of patients at risk are shown below each graph. Abbreviations as in Table II.

cause mortality. In the present study, mean hemoglobin was $12.4 \pm 2.2 \mathrm{~g} / \mathrm{dL}$ in men and $11.8 \pm 1.7 \mathrm{~g} / \mathrm{dL}$ in women. ROC curve analysis for hemoglobin was used in both males and females. The respective ROC cut-off values, yielding maximum sensitivity and specificity, were found at a cut-off point of 12.0 $\mathrm{g} / \mathrm{dL}$ for both the male and female groups, as well as for the overall population (sensitivity $60.8 \%$, specificity $56.2 \%, P<$ 0.001 , area under the curve $=0.61$ ). Comparison of the C-statistics showed a value of 0.61 for age, 0.53 for gender, and 0.81 for postoperative troponin-I. Postoperative troponin-I performed significantly better $(P<0.001)$ as compared with age and gender.

There was a dose-response relationship between the cardiac troponin-I concentration and 6-month mortality (Figure 4). Peak cardiac troponin-I levels were $<0.10 \mathrm{ng} / \mathrm{mL}$ in $87.7 \%(n$ $=658)$, between 0.10 and $1.00 \mathrm{ng} / \mathrm{mL}$ in $7.1 \%(n=53)$, between 1.10 and $2.00 \mathrm{ng} / \mathrm{mL}$ in $2.7 \%(n=20)$, and $>2.10 \mathrm{ng} /$ $\mathrm{mL}$ in $2.5 \%(n=19)$ of the patients. Six-month mortality rates in these groups were $9 \%, 19 \%, 20 \%$, and $58 \%$, respectively. Cardiac troponin-I $>0.10 \mathrm{ng} / \mathrm{mL}$ was associated with a significantly increased risk for 6-month mortality compared with the lowest level group.

\section{DiscuSSION}

The purpose of this study was to evaluate the value of troponin-I in long-term mortality after major noncardiac surgery in practice. The major findings of the study were as follows: 1) Patients with troponin-I elevation after major noncardiac surgery had a poor prognosis in the 7-year follow-up period. This result was mainly derived from the difference within the first 6 months. Moreover, we observed a dose-re-

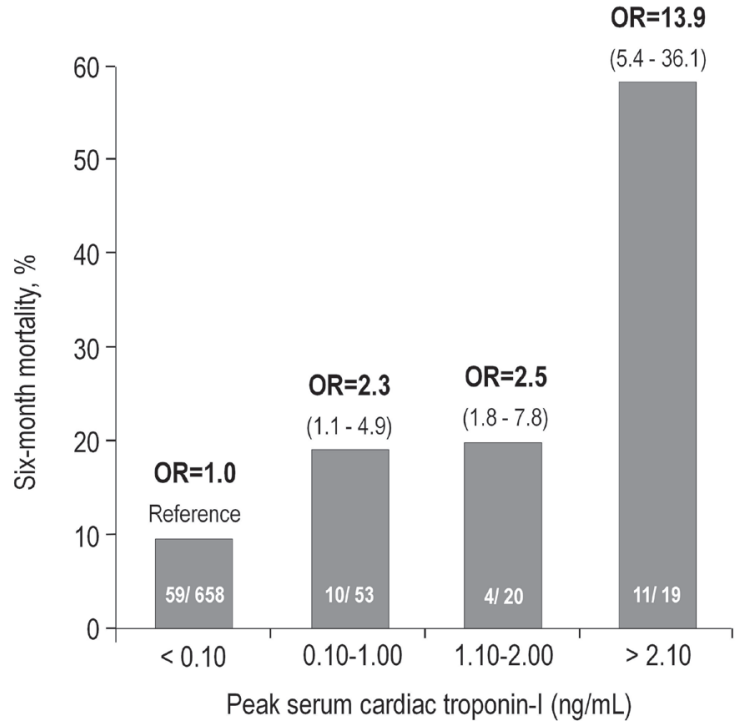

Figure 4. Dose-response relation between cardiac troponin-I concentration and 6-month mortality. Peak cardiac troponin-I concentrations are categorized into 4 groups and presented with 6-month morality rates. Numbers inside bars indicate number of deaths divided by total number of patients in each group. Odds ratios and $95 \%$ confidence intervals for mortality are shown above bars. The $<0.10 \mathrm{ng} / \mathrm{mL}$ group is the reference category to which the other groups were compared.

sponse relationship between the cardiac troponin-I concentration and 6-month mortality. There were no differences in mortality beyond 6 months. 2) Postoperative troponin-I had better prognostic accuracy than age or gender for all-cause death. Age over 70 years, male gender, lower ejection fraction less than $50 \%$, lower hemoglobin level below $12 \mathrm{~g} / \mathrm{dL}$, and resting heart rate over 80 beats per minute were independent predictors of long-term mortality after major noncardiac surgery. However, these thresholds of heart rate, age, and hemoglobin in our study were of little use in predicting all-cause mortality considering a sensitivity of about $50 \%$.

Cardiac troponin testing is a key diagnostic element for the diagnosis and management of patients with acute coronary syndromes. Both cardiac-specific troponin-I and troponin-T are specific and sensitive biomarkers of myocardial damage. ${ }^{11)}$ Direct comparison of the performance of cardiac-specific troponin-I and troponin-T revealed small differences in patients with ACS, but potentially important differences that may be amplified in patients with chronic renal failure. ${ }^{13,14)}$ In previous studies, abnormal levels of cardiac biomarkers were proven to be statistically significant independent predictors of intermediate and long-term outcomes of cardiac events. ${ }^{4,6,8-10)}$ Our study showed that a postoperative troponin-I level above $0.10 \mathrm{ng} / \mathrm{mL}$ was an independent factor for long-term mortality. It is notable that there were significant differences between the TnP and $\mathrm{TnN}$ groups in the rate of all-cause death until 6 months. The 6-month all-cause mortality rate after major noncardiac surgery in patients with $\mathrm{TnP}$ and $\mathrm{TnN}$ was $27.1 \%$ and $8.5 \%$, respectively. This finding was consistent with that of previous reports. ${ }^{9,15)}$ However, these differences were no longer significant after 6 months. This may be because stress due to noncardiac surgery might not last over 6 months, ${ }^{9,15)}$ and most patients that had fatal perioperative MI may die within the first 6 months. ${ }^{16}$ 
Previous studies have suggested that the first 48 to 72 postoperative hours have the highest risk for cardiac complications after major vascular surgery. ${ }^{17,18)}$ Therefore, as suggested previously, the introduction of postoperative monitoring of troponin as a standard protocol might be feasible. ${ }^{19)}$

Troponin-I elevation can occur after noncardiac surgery in patients without coronary artery disease. In one angiographic study, nearly 50 percent of patients with perioperative acute coronary syndrome had no evidence of plaque rupture. ${ }^{20)}$ Perioperative catecholamine changes and hypercoagulable status were present during the postoperative period. ${ }^{21,22)}$ The development of hypertension, hypotension, or tachycardia during surgery may lead to a myocardial oxygen supply-demand mismatch. In addition, the anemia due to surgical bleeding may cause troponin elevation. Thus, an elevated troponin level after noncardiac surgery may be a finding that represents the likely occurrence of type $2 \mathrm{MI}$.

The results of our evaluation of the data are comparable to those of recent trials. ${ }^{6,15,19)}$ The present cohort study estimated the incidence of troponin-I elevation after major noncardiac surgery, and troponin-I positivity above $0.10 \mathrm{ng} / \mathrm{mL}$ was found in $12.2 \%$ of the patients. Studies with vascular and other highrisk but elective surgery showed an incidence of postoperative troponin elevation of 10 to 45 percent. $^{23-26)}$ The incidence of perioperative MI, as found in the present study, was $3.1 \%$, which was not comparable to the recent observational study. ${ }^{19)}$ With regard to the definition, the incidence of perioperative MI is directly related to the sensitivity of the biomarker used. ${ }^{11,15}$ ) Recently, the study by Weber, et al suggested that high-sensitive troponin assay provides strong prognostic information in patients undergoing noncardiac surgery incremental to the widely accepted revised cardiac index. ${ }^{27)}$

Many trials have sought prognosticating factors in noncardiac surgery. ${ }^{4,5,6,10,21,22,27-42)}$ In these studies, poor functional capacity, recent MI and unstable angina, decompensated heart failure, significant arrhythmia, diabetes mellitus, renal insufficiency, cerebrovascular disease, advanced age, tachycardia, anemia, surgical kind, and elevated troponin-I level were determined to be independent prognostic factors after noncardiac surgery, most of which were in agreement with our study. While the results of those studies had been generated after short-term follow-up, the present study extended the follow-up period to 7 years and attempted to provide valuable data regarding longer term mortality. To the best of our knowledge, this study included the longest registry of patients undergoing major noncardiac surgery. Because the present study had complete follow-up in all subjects using data from the National Statistical Office of Korea, our results would be more directly applicable to routine clinical practice and may provide important insight into different clinical courses between early and late follow-up periods. In addition, patients with preexisting troponin-I elevations were excluded, because all-cause mortality can be influenced by myocardial injury which may already have occurred before major noncardiac surgery. ${ }^{6}$ Therefore, the present study focused on postoperative troponin-I at higher levels, and could exclude the effect of preexisting troponin-I elevations.

There are several limitations to our study that should be addressed. First, the present study was conducted at a single center and employed a retrospective design. Therefore, the major limitation was possible selection bias. The high prevalence of DM and CKD may be the result of selection bias, therefore, our cohort was a relatively high-risk population. The second major limitation was the lack of information regarding causespecific mortality in individual patients. As a result, this study could not demonstrate the clinical relevance of troponin-I level in association with cardiac death after noncardiac surgery. We attempted to overcome this limitation by conducting follow-up for all patients. Third, because troponin-I was measured during the first 2 days after and before noncardiac surgery, myocardial damage that may have occurred after that time interval was missed. Previous research has shown that myocardial injury may occur on postoperative day $3 .{ }^{27)}$ Furthermore, the troponin assay used was a relatively old one in a time of high sensitive troponin measures. Fourth, adverse events during noncardiac surgery were not considered, which may have affected operative mortality and all-cause mortality.

Conclusion: Patients with troponin-I elevation after major noncardiac surgery had higher mortality during the 7-year follow-up period, which was mainly derived from the result within the first 6 months. However, there were no differences in mortality beyond 6 months.

\section{Disclosure}

Statement of competing interests: The authors state there are no competing interests to declare.

\section{REFERENCES}

1. Fleisher LA, Beckman J. Anesthesia and noncardiac Surgery in patients with heart disease. In: Bonow RO, L. MD, Zipes DP, Libby P, eds. Braunwald's Heart Disease - A Textbook of Cardiovascular Medicine. 9th ed. Philadelphia, PA: Saunders Elsevier; 2011: 1811-28.

2. Ghaferi AA, Birkmeyer JD, Dimick JB. Variation in hospital mortality associated with inpatient surgery. N Engl J Med 2009; 361: 1368-75.

3. Falahati A, Sharkey SW, Christensen D, et al. Implementation of serum cardiac troponin I as marker for detection of acute myocardial infarction. Am Heart J 1999; 137: 332-7.

4. Lucreziotti S, Foroni C, Fiorentini C. Perioperative myocardial infarction in noncardiac surgery: the diagnostic and prognostic role of cardiac troponins. J Intern Med 2002; 252: 11-20. (Review)

5. Mills NL, Churchhouse AM, Lee KK, et al. Implementation of a sensitive troponin I assay and risk of recurrent myocardial infarction and death in patients with suspected acute coronary syndrome. JAMA 2011; 305: 1210-6.

6. Nagele P, Brown F, Gage BF, et al. High-sensitivity cardiac troponin $\mathrm{T}$ in prediction and diagnosis of myocardial infarction and long-term mortality after noncardiac surgery. Am Heart J 2013; 166: 325-32.

7. Devereaux PJ, Goldman L, Cook DJ, Gilbert K, Leslie K, Guyatt GH. Perioperative cardiac events in patients undergoing noncardiac surgery: a review of the magnitude of the problem, the pathophysiology of the events and methods to estimate and communicate risk. CMAJ 2005; 173: 627-34. (Review)

8. Devereaux PJ, Xavier D, Pogue J, et al. Characteristics and shortterm prognosis of perioperative myocardial infarction in patients undergoing noncardiac surgery: a cohort study. Ann Intern Med 2011; 154: 523-8.

9. Lopez-Jimenez F, Goldman L, Sacks DB, et al. Prognostic value of cardiac troponin $\mathrm{T}$ after noncardiac surgery: 6-month follow-up data. J Am Coll Cardiol 1997; 29: 1241-5. 
10. Levy M, Heels-Ansdell D, Hiralal R, et al. Prognostic value of troponin and creatine kinase muscle and brain isoenzyme measurement after noncardiac surgery: a systematic review and metaanalysis. Anesthesiology 2011; 114: 796-806. (Review)

11. Thygesen K, Alpert JS, White HD, et al. Universal definition of myocardial infarction. Circulation 2007; 116: 2634-53.

12. Maxwell BG, Wong JK, Kin C, Lobato RL. Perioperative outcomes of major noncardiac surgery in adults with congenital heart disease. Anesthesiology 2013; 119: 762-9.

13. Rubini Gimenez M, Twerenbold R, Reichlin T, et al. Direct comparison of high-sensitivity-cardiac troponin I vs. T for the early diagnosis of acute myocardial infarction. Eur Heart J 2014; 35: 2303-11.

14. Giannitsis E, Katus HA. Comparison of cardiac troponin T and troponin I assays--implications of analytical and biochemical differences on clinical performance. Clin Lab 2004; 50: 521-8. (Review)

15. Kim LJ, Martinez EA, Faraday N, et al. Cardiac troponin I predicts short-term mortality in vascular surgery patients. Circulation 2002; 106: 2366-71.

16. Peduzzi P, Detre K, Murphy ML, Thomsen J, Hultgren H, Takaro T. Ten-year incidence of myocardial infarction and prognosis after infarction. Department of Veterans Affairs Cooperative Study of Coronary Artery Bypass Surgery. Circulation 1991; 83: 747-55.

17. Longhitano $\mathrm{S}$, Coriat $\mathrm{P}$, Agrò F. Postoperative myocardial infarction: pathophysiology, new diagnostic criteria, prevention. Minerva Anestesiol 2006; 72: 965-83. (Review, English, Italian)

18. Le Manach Y, Perel A, Coriat P, Godet G, Bertrand M, Riou B. Early and delayed myocardial infarction after abdominal aortic surgery. Anesthesiology 2005; 102: 885-91.

19. van Waes JA, Nathoe HM, de Graaff JC, et al. Myocardial injury after noncardiac surgery and its association with short-term mortality. Circulation 2013; 127: 2264-71.

20. Gualandro DM, Campos CA, Calderaro D, et al. Coronary plaque rupture in patients with myocardial infarction after noncardiac surgery: frequent and dangerous. Atherosclerosis 2012; 222: 191-5.

21. Sametz W, Metzler H, Gries M, et al. Perioperative catecholamine changes in cardiac risk patients. Eur J Clin Invest 1999; 29: 582-7.

22. Parker SD, Breslow MJ, Frank SM, et al. Catecholamine and cortisol responses to lower extremity revascularization: correlation with outcome variables. Perioperative Ischemia Randomized Anesthesia Trial Study Group. Crit Care Med 1995; 23: 1954-61.

23. McIlroy DR, Chan MT, Wallace SK, et al. Automated preoperative assessment of endothelial dysfunction and risk stratification for perioperative myocardial injury in patients undergoing noncardiac surgery. Br J Anaesth 2014; 112: 47-56.

24. Lucreziotti S, Conforti S, Carletti F, et al. Cardiac troponin-I elevations after thoracic surgery. Incidence and correlations with baseline clinical characteristics, C-reactive protein and perioperative parameters. Rev Esp Cardiol 2007; 60: 1159-66. (Spanish)

25. Blecha MJ, Clark ET, Worley TA, Salazar MR, Podbielski FJ. Predictors of electrocardiographic change, cardiac troponin elevation, and survival after major vascular surgery: a community hospital experience. Am Surg 2007; 73: 697-702.

26. Ausset S, Auroy Y, Lambert E, et al. Cardiac troponin I release after hip surgery correlates with poor long-term cardiac outcome. Eur J Anaesthesiol 2008; 25: 158-64.

27. Weber M, Luchner A, Seeberger M, et al. Incremental value of high-sensitive troponin $\mathrm{T}$ in addition to the revised cardiac index for peri-operative risk stratification in non-cardiac surgery. Eur Heart J 2013; 34: 853-62.

28. Carson JL, Duff A, Poses RM, et al. Effect of anaemia and cardiovascular disease on surgical mortality and morbidity. Lancet 1996;
348: 1055-60.

29. Lee TH, Marcantonio ER, Mangione CM, et al. Derivation and prospective validation of a simple index for prediction of cardiac risk of major noncardiac surgery. Circulation 1999; 100: 1043-9.

30. Poldermans D, Boersma E, Bax JJ, et al. The effect of bisoprolol on perioperative mortality and myocardial infarction in high-risk patients undergoing vascular surgery. Dutch Echocardiographic Cardiac Risk Evaluation Applying Stress Echocardiography Study Group. N Engl J Med 1999; 341: 1789-94.

31. Reilly DF, McNeely MJ, Doerner D, et al. Self-reported exercise tolerance and the risk of serious perioperative complications. Arch Intern Med 1999; 159: 2185-92.

32. Boersma E, Poldermans D, Bax JJ, et al. Predictors of cardiac events after major vascular surgery: Role of clinical characteristics, dobutamine echocardiography, and beta-blocker therapy. JAMA 2001; 285: 1865-73.

33. Fleisher LA, Eagle KA. Clinical practice. Lowering cardiac risk in noncardiac surgery. N Engl J Med 2001; 345: 1677-82. (Review)

34. Girish M, Trayner E Jr, Dammann O, Pinto-Plata V, Celli B. Symptom-limited stair climbing as a predictor of postoperative cardiopulmonary complications after high-risk surgery. Chest 2001; 120: 1147-51.

35. Mann JF, Gerstein HC, Pogue J, Bosch J, Yusuf S. Renal insufficiency as a predictor of cardiovascular outcomes and the impact of ramipril: the HOPE randomized trial. Ann Intern Med 2001; 134 : 629-36.

36. Best PJ, Lennon R, Ting HH, et al. The impact of renal insufficiency on clinical outcomes in patients undergoing percutaneous coronary interventions. J Am Coll Cardiol 2002; 39: 1113-9.

37. Stevens RD, Burri H, Tramèr MR. Pharmacologic myocardial protection in patients undergoing noncardiac surgery: a quantitative systematic review. Anesth Analg 2003; 97: 623-33. (Review)

38. Shander A. Anemia in the critically ill. Crit Care Clin 2004; 20: 159-78. (Review)

39. Fleisher LA, Beckman JA, Brown KA, et al. ACC/AHA 2007 guidelines on perioperative cardiovascular evaluation and care for noncardiac surgery: a report of the American College of Cardiology/American Heart Association Task Force on Practice Guidelines (Writing Committee to Revise the 2002 Guidelines on Perioperative Cardiovascular Evaluation for Noncardiac Surgery) developed in collaboration with the American Society of Echocardiography, American Society of Nuclear Cardiology, Heart Rhythm Society, Society of Cardiovascular Anesthesiologists, Society for Cardiovascular Angiography and Interventions, Society for Vascular Medicine and Biology, and Society for Vascular Surgery. J Am Coll Cardiol 2007; 50: e159-241.

40. Dunkelgrun M, Boersma E, Schouten O, et al. Bisoprolol and fluvastatin for the reduction of perioperative cardiac mortality and myocardial infarction in intermediate-risk patients undergoing noncardiovascular surgery: a randomized controlled trial (DECREASE-IV). Ann Surg 2009; 249: 921-6.

41. Fleisher LA, Beckman JA, Brown KA, et al. 2009 ACCF/AHA focused update on perioperative beta blockade incorporated into the ACC/AHA 2007 guidelines on perioperative cardiovascular evaluation and care for noncardiac surgery: a report of the American college of cardiology foundation/American heart association task force on practice guidelines. Circulation 2009; 120: e169276.

42. van Diepen S, Bakal JA, McAlister FA, Ezekowitz JA. Mortality and readmission of patients with heart failure, atrial fibrillation, or coronary artery disease undergoing noncardiac surgery: an analysis of 38047 patients. Circulation 2011; 124: 289-96. 